\title{
Dysphagia in Wallenberg's Syndrome: The Challenge in Treatment
}

\author{
Prabhjot Kaur* \\ Max Super Specialty Hospital, Saket, India
}

Submission: October 25, 2016; Published: October 27, 2016

*Corresponding author: Prabhjot Kaur, Max Super Specialty Hospital, Saket, India, Tel: 91-9971884421; Email: prabhjot.bkp@gmail.com

\begin{abstract}
Wallenberg's syndrome (WS) has been observed as the cause of most severe form of dysphagia among all varieties of stroke. Although dysphagia resolves in most cases within 1-2 months, it may persist for months or years altogether resulting in malnutrition, dehydration and other complications. Rostral lesions lead to these complications and it tends to involve not only the oropharyngeal phase, but the esophageal phase as well. The interventions recommended include techniques and maneuvers along with use of Transcranial Magnetic Stimulation and Neuro Muscular Electrical Stimulation.
\end{abstract}

Keywords: Wallenberg's syndrome; Dysphagia; Lateral medullary stroke; Aspiration

\section{Introduction}

Wallenberg syndrome was initially described by Gaspard Vieusseaux of Geneva in 1810. It was characterized by vertigo, unilateral facial numbness, loss of pain and temperature appreciation in the opposite limbs, dysphagia and hoarseness, minor tongue involvement, hiccups and a dropped eyelid [1] Wallenberg described in his case reports from 1895-1922 how the patients could not swallow solids at all while liquids were taken in minute amounts [2]. In 2003, Kim [3] described findings in WS based on clinical MRI in a correlation study on 130 patients. There was sudden onset of WS in $75 \%$ cases and it typically started with headache, vertigo, dizziness, and gait ataxia. The symptoms that appeared later include dysphagia, hoarseness, hiccups, and sensory signs. Among these, dysphagia was found in $65 \%$ cases [3]. Although cerebral, cerebellar, or brainstem strokes can all impair swallowing physiology, brainstem strokes can result in the largest swallowing compromise despite they are less common than cortical lesions. Brainstem lesions can affect sensation of the mouth, tongue and cheek, timing in the trigger of the pharyngeal swallow, laryngeal elevation, glottic closure, and cricopharyngeal relaxation [4].

Kwon et al. [5] studied 46 patients (37 with WS and 9 with medial medullary infarct - MMI) based on video fluoroscopy. They found that MMI caused problem in timing of hyolaryngeal elevation whereas excursion of hyolaryngeal elevation was affected in WS [5]. Dysphagia in WS, which is commonly associated with diminished gag reflex, has been attributed to affected efferent fibres of cranial nerves IX and X, and nucleus ambiguus (NA). It is more commonly found in rostral lesions. No significant motor $\mathrm{V}$ or XII nerve disturbances occur in WS. $\mathrm{Na}$ involvement results in ipsilateral vocal cord paralysis and ipsilateral palate weakness. Unilateral WS produces bilateral pharyngeal and laryngeal dysfunction making the dysphagia severe based on electrophysiological evidence [6]. However, based on stydu by Kim $\mathrm{H}$ et al. [7] on 23 WS patients, no aspiration occurs with lower level medullary lesions. Swallowing is a patterned sequence of motor activities that result from bilateral input from swallowing centres in rostral dorsolateral medulla (Nucleus Tractus Solitarius - NTS, NA, and reticular formation). These central pattern generators of deglutition are impaired in WS leading to disturbed sequential muscle activity during oropharyngeal phase with incoordination.

The controversy regarding presence of pure oropharyngeal or oropharyngeal with esophageal dysphagia in WS was settled by Longemann et al. [8]. They found that cricopharyngeal muscle and striated portion of esophagus are spastic in WS affecting upper esophageal sphincter (UES) function [8-10]. Also, Robbins \& Levine [2] found impaired cricopharyngeal relaxation in WS patient. They concluded if WS dysphagia was due to oropharyngeal deficits alone, then extensive therapy with manoeuvres should have been sufficient to pass bolus through upper esophageal sphincter [2]. It has been widely accepted 


\section{Open Access Journal of Neurology \& Neurosurgery}

that in most cases, the dysphagia is severe enough to require nasogastric (NG) feeding but often improves rapidly, and the patient can return to oral feeding within 1-2 months after the stroke [6]. Martino et al. [11] performed MBSS (modified barium swallow study) at $3,9,14$, and 27 months post WS. Their findings were:

- $\quad$ At 3 months, normal AP propulsion, absent pharyngeal swallow.

- At 9 months, anterior and vertical elevation of larynx slightly decreased, epiglottic deflection present, no contraction of middle and inferior pharyngeal constrictors, UES failed to open, bolus pooled in hypopharynx.

- At 14 months, swallows effective with liquids and soft solids.

- $\quad$ At 27 months, more consistent epiglottic deflection for airway protection, impaired laryngeal phase.

The interventions recommended for WS dysphagia have concentrated on large bolus volume ( increases pharyngeal wall movement, duration of hyolaryngeal excursion, duration of UES opening, width of UES opening) [9], head turn to weaker side (increases range of hyolaryngeal movement) [5], Mendelsohn manoeuvre (increases duration of UES opening), shaker exercise ( improves diameter of UES opening) [12], etc. Shaker exercise has been found useful in cases with aspiration [13]. Also used for WS dysphagia treatment are techniques like Transcranial Magnetic Stimulation (TMS) and Neuromuscular Electrical Stimulation (NMES).

Evidence related to treatment of WS dysphagia has been scarce keeping in mind that it leads to the most severe form of dysphagia among all strokes. It can be managed efficiently with focus on both its oropharyngeal and esophageal components. This could reduce the chance of aspiration with early detection being the mainstay of treatment.

\section{References}

1. Pearce JM (2000) Wallenberg syndrome. J Neurol Neurosurg Psychiatry 68(5): 570.

2. Robbins J, Levine $R$ (1993) Swallowing after lateral medullary syndrome plus. Clinics in communication disorders 3(4): 45-55.

3. Kim JS (2003) Pure lateral medullary stroke: clinical- radiological correlation of 130 acute, consecutive patients. Brain 126(8): 18641872 .

4. Martino R, Foley N, Bhogal S, Diamant N, Speechley M, et al. (2005) Dysphagia after stroke: incidence, diagnosis, and pulmonary complications. Stroke 36(12): 2756-2763.

5. Kwon M, Lae JH, Kim JS (2005) Dysphagia in unilateral medullary infarction: lateral vs. Medial lesions. Neurology 65(5): 714-718.

6. Aydogdu I, Ertekin C, Tarleci S, Turman B, Kiylioglu N, et al. (2001) Dysphagia in lateral medullary syndrome: an acute disconnection syndrome in premotor neurons related to swallowing activity. Stroke 32(9): 2081-2087.

7. Kim H, Ching CS, Lee KH, Robbins J (2000) Aspiration subsequent to a pure medullary infarction. Archives of neurology 57(4): 478-483.

8. Longemann JA (1998) Evaluation and treatment of swallowing disorders. ( $2^{\text {nd }}$ edn), Austin, Texas, USA.

9. Jacob P, Kahrilas P, Longemann JA, Shah V, Ha T (1989) Upper esophageal sphincter opening and modulation during swallowing. Gastroenterology 97(6): 469-478.

10. Vigderman AM, Chavin JM, Kososky C, Tahmoush AJ (1998) Aphagia due to pharyngeal constrictor paresis from lateral medullary infarction. J Neurol Sci 155(2): 208-210.

11. Martino R, Terrault N, Ezerzer F, Mikulis D (2001) Dysphagia in patients with lateral medullary syndrome: insight into the central control of swallowing. Gastroenterology 121(2): 420-426.

12. Shaker R, Easterling C, Kern M, Nitschke T, Massey B, et al. (2002) Rehabilitation of swallowing by exercise in tube-fed patients with pharyngeal dysphagia secondary to abnormal upper esophageal sphincter opening. Gastroeneterology 122(5): 1314-1321.

13. Longemann J A, Rademaker A, Pauloski B R, Kelly A, et al. (2009) A randomized study comparing the shaker exercise with traditional therapy: a preliminary study. Dysphagia 24(4): 403-411.

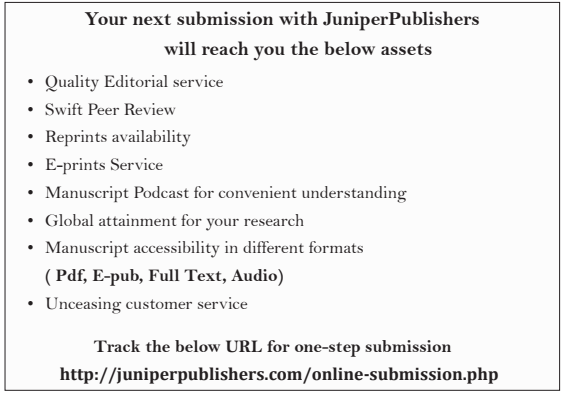

